\title{
Fulvene-5 potently inhibits NADPH oxidase 4 and blocks the growth of endothelial tumors in mice
}

Sulochana S. Bhandarkar, ${ }^{1}$ Marisa Jaconi, ${ }^{2}$ Levi E. Fried, ${ }^{1}$ Michael Y. Bonner, ${ }^{1}$ Benjamin Lefkove, ${ }^{1}$ Baskaran Govindarajan, ${ }^{1}$ Betsy N. Perry, ${ }^{1}$ Ravi Parhar, ${ }^{1}$ Jamie Mackelfresh, ${ }^{1}$ Allie Sohn, ${ }^{1}$ Michael Stouffs, ${ }^{2}$ Ulla Knaus, ${ }^{3}$ George Yancopoulos, ${ }^{4}$ Yvonne Reiss, ${ }^{5}$ Andrew V. Benest, ${ }^{6}$ Hellmut G. Augustin, ${ }^{6}$ and Jack L. Arbiser ${ }^{1}$

\begin{abstract}
${ }^{1}$ Department of Dermatology and Atlanta Veterans Administration Medical Center, Emory University, Atlanta, Georgia, USA. 2Department of Pathology and Immunology, Faculty of Medicine, Geneva University, Geneva, Switzerland.

${ }^{3}$ Department of Immunology, IMM28, The Scripps Research Institute, La Jolla, California, USA. ${ }^{4}$ Department of Vascular Biology and Angiogenesis Research, Regeneron Pharmaceuticals Inc., Tarrytown, New York, USA. Institute of Neurology (Edinger Institute), Frankfurt University Medical School, Frankfurt, Germany. ${ }^{6}$ Joint Research Division Vascular Biology, Medical Faculty Mannheim, Heidelberg University (CBTM), and German Cancer Research Centre Heidelberg (DKFZ-ZMBH Alliance), Heidelberg, Germany.
\end{abstract}

\begin{abstract}
Hemangiomas are the most common type of tumor in infants. As they are endothelial cell-derived neoplasias, their growth can be regulated by the autocrine-acting Tie2 ligand angiopoietin 2 (Ang2). Using an experimental model of human hemangiomas, in which polyoma middle $T$-transformed brain endothelial (bEnd) cells are grafted subcutaneously into nude mice, we compared hemangioma growth originating from bEnd cells derived from wild-type, Ang2 ${ }^{+/}$, and Ang $2^{-/-}$mice. Surprisingly, Ang2-deficient bEnd cells formed endothelial tumors that grew rapidly and were devoid of the typical cavernous architecture of slow-growing Ang2-expressing hemangiomas, while $\mathrm{Ang} 2^{+/-}$cells were greatly impaired in their in vivo growth. Gene array analysis identified a strong downregulation of NADPH oxidase 4 (Nox4) in $A n g 2^{+/-}$cells. Correspondingly, lentiviral silencing of Nox4 in an Ang2-sufficient bEnd cell line decreased Ang2 mRNA levels and greatly impaired hemangioma growth in vivo. Using a structure-based approach, we identified fulvenes as what we believe to be a novel class of Nox inhibitors. We therefore produced and began the initial characterization of fulvenes as potential Nox inhibitors, finding that fulvene-5 efficiently inhibited Nox activity in vitro and potently inhibited hemangioma growth in vivo. In conclusion, the present study establishes Nox 4 as a critical regulator of hemangioma growth and identifies fulvenes as a potential class of candidate inhibitor to therapeutically interfere with Nox function.
\end{abstract}

\section{Introduction}

Hemangiomas are the most common neoplasms of infancy. While the exact pathogenesis of these lesions remains obscure, prior studies have shown that they are clonal neoplasms (1). These lesions, unlike most other neoplasms, undergo spontaneous regression. The molecular repertoire of angiogenic cytokines controlling hemangioma growth is poorly understood, but evidence has emerged that the Tie2 ligand angiopoietin 2 (Ang2) plays a dominant role in hemangioma growth $(1,2)$. While hemangiomas of infancy are exclusively a disorder of childhood, a histologically similar lesion is acquired through infection by the bacterium Bartonella bacilliformis in adults. B. bacilliformis infection similarly leads to increased production of Ang2 (3).

We have previously shown that pharmacologic blockade of Ang2 in a murine model of hemangiomas leads to decreased growth in vivo (2). We therefore expected that polyoma middle T oncogene-transformed endothelial cells derived from Ang2-deficient

Conflict of interest: A patent is pending on fulvene-5, with J.L. Arbiser as inventor and Emory University as owner of the patent.

Nonstandard abbreviations used: Ang2, angiopoietin 2; bEnd, brain endothelial DLL4, delta-like ligand 4; Nox4, NADPH oxidase 4; Nrarp, Notch-related ankyrin repeat protein; PLGF, placental growth factor.

Citation for this article: J. Clin. Invest. 119:2359-2365 (2009). doi:10.1172/JCI33877. mice would be unable to form hemangiomas in vivo. Surprisingly, Ang2-deficient endothelioma cells formed tumors much faster than Ang2-expressing endothelioma cells, but these tumors were not hemangiomas; rather, they histologically resembled angiosarcomas. We therefore hypothesized that endothelioma-derived Ang2 may control critical downstream mediators of hemangioma growth. Exploratory gene array experiments identified the NADPH oxidase Nox4 as being downregulated in Ang2 heterozygous cells that grow poorly in vivo. Thus, loss of Ang2 appears to affect the ability of endothelial cells to form hemangiomas, while loss of Nox 4 appears to have a more severe phenotype, in significantly inhibiting the growth of hemangiomas in vivo. We also demonstrate that novel fulvenes, which block Nox4, have potent inhibitory effects on hemangioma growth in vivo.

\section{Results}

Ang2 $2^{--}$cells form rapidly growing tumors in vivo while Ang2 $2^{+-}$and Ang2 $2^{++}$form tumors with greatly delayed latency. Polyoma middle $\mathrm{T}$-transformed endothelial cells were established from primary endothelial cells isolated from P3 murine brains of wild-type, Ang2-heterozygous, and Ang2-deficient mice. In order to study the tumor-forming potential of these cells, low numbers of $A n g 2^{-/-}$, Ang $2^{+/-}$, and $A n g 2^{+/+}$cells $\left(2.5 \times 10^{5}\right)$ were injected subcutaneously into nude mice. The mice were observed over a period of 4 weeks. 


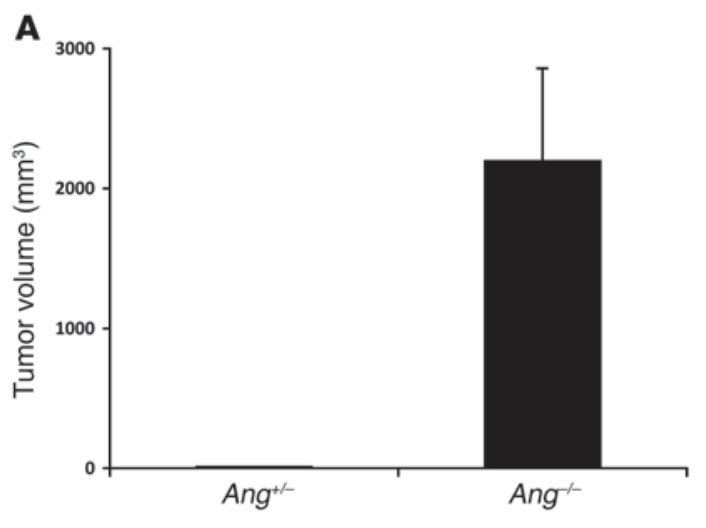

$\mathbf{K}$

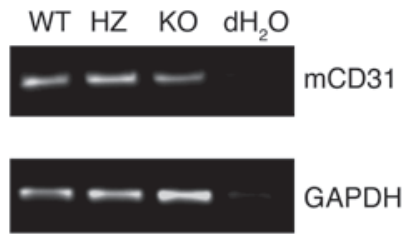

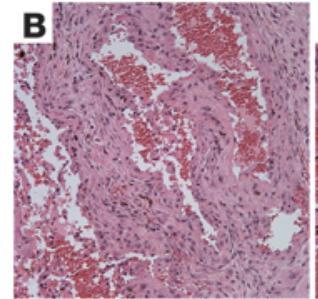
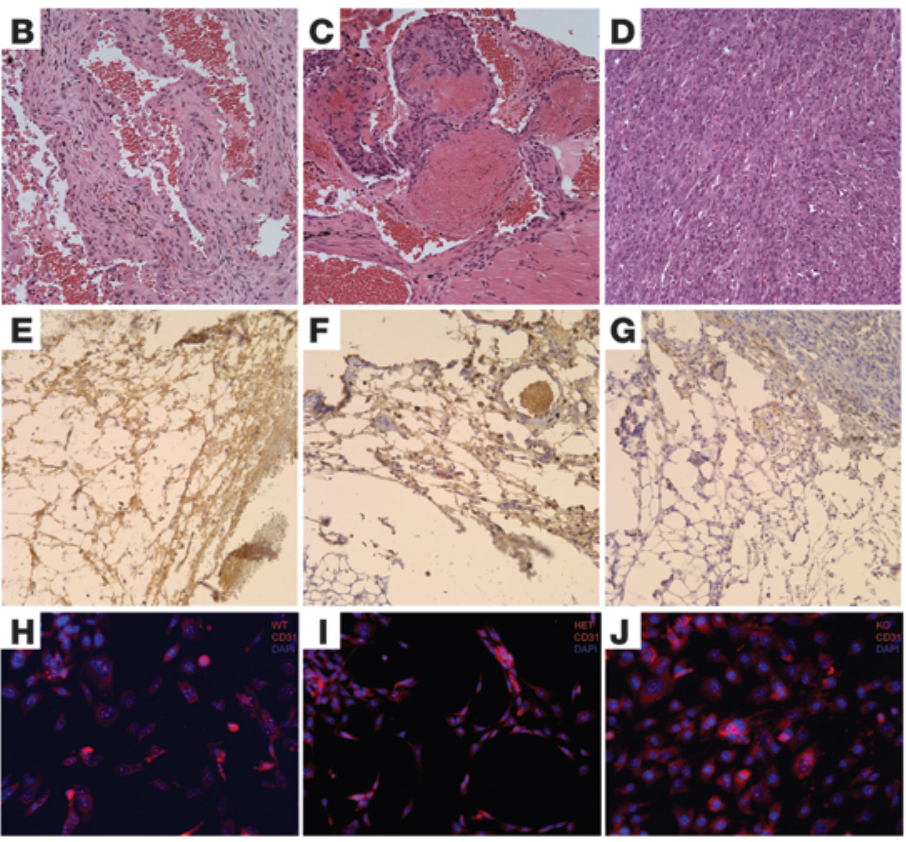

\section{Figure 1}

Differential tumor growth in endotheliomas derived from Ang2 wild-type, heterozygous, and knockout mice. Tumor formation in Ang2 $2^{-/-}$cells and Ang $2^{+/-}$cells in vivo (A). Six mice were injected with $2.5 \times 10^{5} \mathrm{Ang}^{-/-}$or Ang $^{+/-}$cells. Animals were euthanized on day 30 , secondary to tumor burden in the $\mathrm{Ang}^{-/-}$animals. The figure depicts the average tumor volume $\left(\mathrm{mm}^{3}\right)$ in both experimental groups. Error bar represents SEM. $P<0.05$ versus control. Morphological analysis revealed typical cavernous hemangiomas in wild-type endothelioma-derived hemangiomas (B) and in $\mathrm{Ang}^{+/-}$endothelioma-derived hemangiomas (C) compared with the solid growth of $A n g 2^{-/-}$endothelioma-derived sarcomas (D). (E-G) CD31 immunohistochemistry of $A n g 2^{+/+}, A n g 2^{+/}$, and $A n g 2^{-/-}$tumors, respectively, with positive staining in all 3 tumors, but decreased differentiation in the Ang2-/- tumors. (H-J) Immunocytochemistry of CD31, with pink staining representing CD31 and blue DAPI staining representing nuclei. (J) RT-PCR of Ang2 $2^{+/}$, Ang2 $2^{+-}$, and $A n g 2^{-/-}$cells for CD31, with GAPDH as a loading control. Original magnification, $\times 40$. (K) Represented is RT-PCR for CD31 in all 3 phenotypes of endothelial cells. Note presence of the endothelial marker CD31 in all 3 types of cells. GAPDH represents a loading control. PCR was performed using QIAGEN reagents per manufacturer's protocol and 35 cycles performed at an annealing temperature of $55^{\circ} \mathrm{C}$. To confirm expression of the endothelial marker CD31, PCR was performed using the following primer sequences: forward, 5'-GTGAAGGTGCATGGCGTATC-3'; reverse 5'-CACAAAGTTCTCGTTGGAGGT-3', producing a 192-bp product. PCR for GAPDH was used as a control: forward, 5'-AGGTCGGTGTGAACGGATTT-3'; reverse, 5'-CTCCTGGAAGATGGTGATGG-3', resulting in a 224-bp band.

Under these experimental conditions, Ang2 $2^{+/}$and $A n g 2^{+/-}$cells formed slow-growing tumors with the characteristic morphological appearance of cavernous hemangiomas (Figure 1, A-C). In contrast, $A n g 2^{-/-}$cells generated from knockout littermates formed much faster-growing tumors that had lost the typical cavernous hemangioma-like morphology of tumors originating from wild-type cells and resembled angiosarcoma instead (Figure 1D). Immunostaining for CD31, RT-PCR for CD31, and CD31 immunohistochemistry demonstrated that all lesions retained endothelial differentiation, but tumors derived from Ang $2^{-/-}$cells were histologically sarcomatous rather than hemangiomas. In fact, the growth difference was so pronounced that a detailed side-byside comparison of wild-type hemangiomas with heterozygous and Ang $2^{-/-}$hemangiomas was not possible because Ang $2^{-/-}$hemangioma-bearing mice had to be sacrificed prior to the growth of Ang2 $2^{+/-}$or $A n g 2^{+/+}$neoplasms.

Nox4 and Nrarp are strongly downregulated in Ang2-beterozygous endothelial cells. Comparative gene array experiments were performed in order to yield mechanistic insight into the pronounced growth difference between Ang2-deficient and Ang2-heterozygous endothelial cells. Among the differentially expressed genes, Notchrelated ankyrin repeat protein (Nrarp) and Nox4 were identified as among the most differentially expressed. Confirmatory quantita- tive RT-PCR analyses validated the array experiments and demonstrated the pronounced downregulation of Nrarp and Nox 4 expression in Ang2 $2^{+-}$cells compared with Ang2 $2^{-/-}$cells (Figure 2).

bEnd. 3 Nox4shRNA cells show decreased expression of Ang2 and Nox4 in vitro. In order to determine whether Ang 2 and Nox4 expression is associatively linked, Nox4 shRNA-mediated knockdown cells were generated in brain endothelial 3 (bEnd.3) cells, a well-established model of hemangiomas. Knockdown was efficient, with a 91\% decrease in Nox4 expression (Figure 3A) and a concomitant $73 \%$ decrease in Ang2 expression in bEnd.3 Nox4shRNA cells (Figure 3B) compared with control cells.

bEnd. 3 Nox4shRNA cells show reduced tumor growth. In order to determine whether the high tumorigenic potential of Ang2-/- endothelium may be related to dysregulated Nox4 expression, Nox4 expression was shRNA silenced in bEnd. 3 endothelioma cells and the tumorigenicity of Nox4-silenced and control bEnd. 3 cells was studied in vivo. Toward this end, large numbers of bEnd. 3 cells $\left(10^{6}\right)$ were injected subcutaneously into nude male mice and tumor growth was monitored for a period of 2 weeks. Control bEnd. 3 cells readily formed large hemangiomas, whereas Nox4-silenced cells grew only small nodules (Figure 4).

Fulvene-5 inhibits Nox function in vitro. In order to inhibit Nox4 function pharmacologically, we tested fulvene derivatives as a 


\section{A}

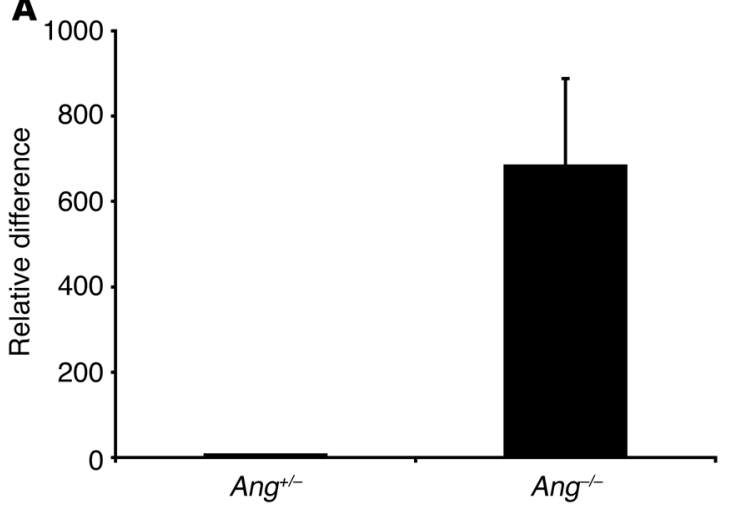

B

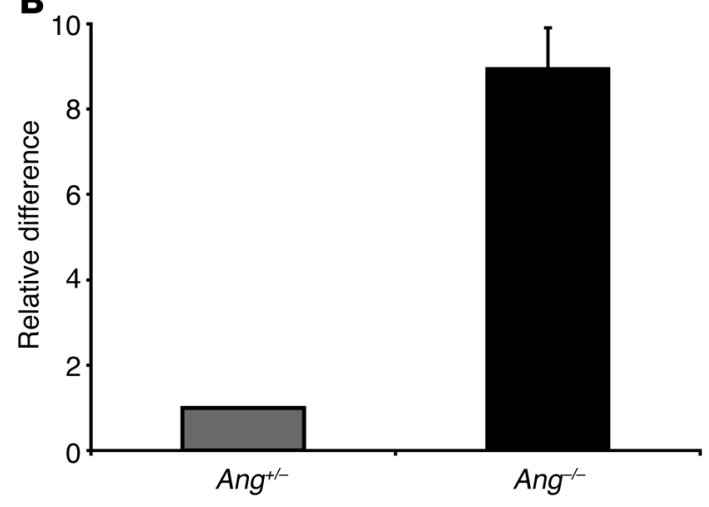

Figure 2

Downregulation of Nrarp and Nox4 mRNA in Ang2 heterozygous endothelioma cells. RT-PCR analysis of Ang2 $2^{-/-}$and Ang2 $2^{+/-}$cells. Nrarp mRNA expression (A) and Nox4 mRNA expression (B) are increased in Ang2 $2^{-/-}$cells compared with Ang2 $2^{+-}$cells. Bars represent the average of triplicate experiments, and error bars indicate SEM. $P<0.05$ versus control.

potentially new class of Nox inhibitors. We used HEK293 cells stably transfected with constitutively active Nox 4 and COSphox cells harboring inducible Nox $2 / \mathrm{p} 47$ phox/p67phox complex and measured hydrogen peroxide generation by homovanillic acid (HVA) assay. Fulvene-5 treatment decreased Nox4- and Nox2-mediated ROS production by approximately $40 \%$ (Figure 5).

Fulvene-5 downregulates Ang2, Nrarp, Dll4, and PlGF expression in $b$ End. 3 cells. We confirmed the decreased expression of Ang2, deltalike ligand 4 (DLL4), placental growth factor (PLGF), and Nrarp in a dose-dependent manner in bEnd. 3 cells treated with fulvene- 5 and determined a $99 \%$ decrease in Nrarp expression, a $60 \%$ decrease in PLGF expression, a 75\% decrease in DLL4 expression, and at the same time, a 25\% decrease in Ang2 expression in bEnd.3 treated with $1.0 \mu \mathrm{M}$ of fulvene-5, consistent with data obtained from either loss of Ang2 or Nox 4 knockdown in polyoma-transformed endothelial cells (Figure 6).

Fulvene-5 reduces hemangioma formation in mice injected with $b$ End. 3 cells. To determine whether fulvene-5-induced loss of Nox4 demonstrated antitumorigenicity, we injected nude mice subcutaneously with high numbers of bEnd. 3 cells $\left(10^{6}\right)$. Mice were treated with fulvene-5
$(120 \mathrm{mg} / \mathrm{kg} / \mathrm{d})$ or vehicle control for 2 weeks, and tumor growth as well as tumor burden were analyzed thereafter. Tumor growth in mice treated with fulvene- 5 was significantly reduced compared with that in vehicle control-treated mice (Figure 7, A and B).

\section{Discussion}

The Tie2 ligand Ang2 has been identified as an antagonistic ligand of constitutive Ang1/Tie2 signaling (4). As such, it destabilizes the vascular endothelium and primes it to respond to exogenous cytokines (5). Yet Ang2 functions are contextual, and Ang2 has been shown to be capable of acting both as an inhibitory ligand of Tie2 and as a stimulating ligand even though the molecular mechanisms controlling agonistic versus antagonistic functions of Ang2 are largely unknown (5). Importantly, Ang2 is almost exclusively produced by endothelial cells themselves $(6,7)$. Since it is produced by its own target cell, it functions as an autocrine-acting growth factor of endothelial cells.

Hemangiomas are endothelial-derived benign vascular neoplasms. Corresponding to its endothelial cell origin, Ang2 has in recent years been identified as a major regulator of heman-
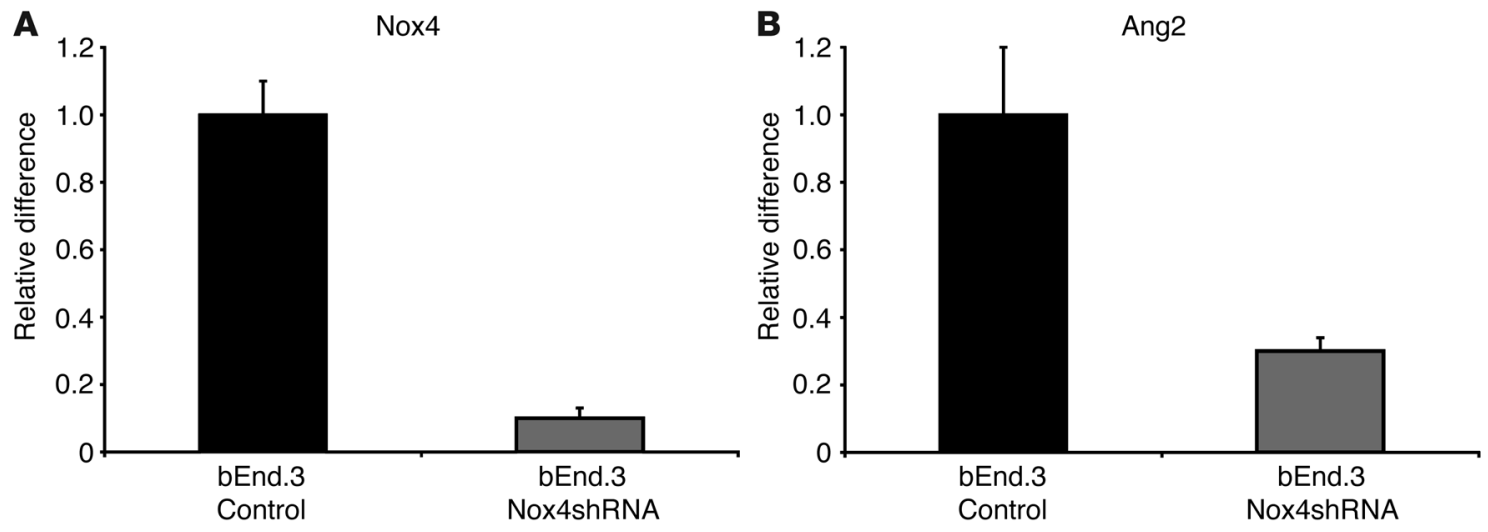

Figure 3

Nox4 siRNA downregulates endogenous Nox4 and Ang2 in bEnd.3 cells. Expression of Nox4 (A) and Ang2 (B) in bEnd.3 Nox4siRNA cells. Nox4 mRNA and Ang2 mRNA expression are decreased in bEnd.3 Nox4shRNA cells. Bars represent the average of triplicate experiments, and error bars indicate SEM. $P<0.05$ versus control. 


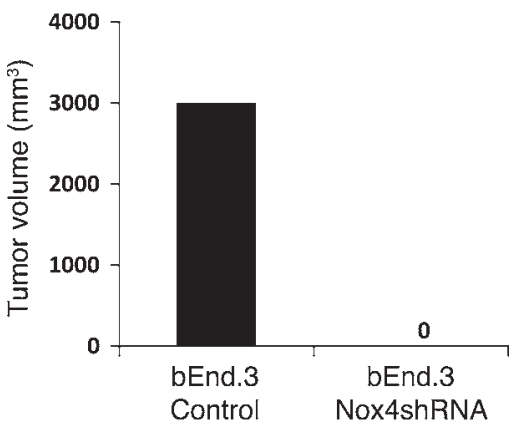

Figure 4

Tumor volumes in bEnd. 3 control versus bEnd. 3 Nox4shRNA. Six mice were injected with $10^{6}$ bEnd.3 control and bEnd.3 Nox4shRNA cells. Animals were euthanized on day 14 , secondary to tumor burden in the bEnd. 3 control animals. The figure depicts average tumor volume $\left(\mathrm{mm}^{3}\right)$ in each group. Error bars represent SEM. $P<0.05$ versus control.

gioma growth $(1,2)$. Based on these findings, the present study was aimed at molecularly dissecting the role of Ang2 during hemangioma growth. The study yielded surprising findings that shed mechanistic insights into the pathogenesis of hemangioma growth and open the door toward modalities that may therapeutically interfere with hemangiomas. Specifically, the present study identifies (a) loss of hemangioma differentiation in tumors derived from Ang2-knockout cells, indicating a requirement for Ang2 in hemangioma genesis; (b) the downregulation of Nox4 in polyoma-transformed endothelial cells in poorly tumorigenic Ang2 $2^{+/-}$cells; (c) reduced hemangioma Ang2 production upon Nox4 silencing; (d) reduced hemangioma growth upon Nox4 silencing; and (e) fulvene-5 as inhibitor of Nox4 and correspondingly as potent inhibitor of hemangioma growth.

NADPH oxidases (Nox) are a family of NADPH-dependent enzymes that generate reactive oxygen species and consist of 7 members in humans (8-13). These enzymes are part of multiunit complexes that may include $\mathrm{p} 22^{\text {phox }}$, cytosolic oxidase components, and the small GTPases Rac1 and Rac2. The first Nox enzyme to be identified was Nox2 (gp91phox), which is frequently mutated in chronic granulomatous disease and is responsible for the phagocyte respiratory burst $(14,15)$. Other Nox/Duox enzymes were discovered as superoxide/hydrogen peroxide-generating enzymes in other cell types. Nox1 is highly expressed in gastrointestinal epithelium, Nox3 in the inner ear, and Nox 4 in a number of tissues including malignant melanoma $(16,17)$. We have previously shown that Nox 4 is expressed in endothelial cells and in bEnd. 3 cells (2).

A role for Nox in hemangioma growth was circumstantially suggested in earlier experiments. We have demonstrated that topical application of eosin, a potent Nox inhibitor, causes regression of ulcerated hemangiomas of infancy (18). Likewise, systemic propranolol, a beta blocker, has also shown efficacy in hemangiomas of infancy (18). Propranolol has activity against reactive oxygen in addition to its activity against the $\beta$-adrenergic receptor (19).

The identification of a critical role of Nox 4 in hemangioma growth suggested that Nox may serve as a target to therapeutically interfere with hemangioma growth. Small molecule inhibitors of Nox have been synthesized, but in vivo data are limited. Based upon structural similarities to the well-studied Nox inhibitor diphenylene iodonium, we found that triphenylmethane dyes are potent inhibitors of Nox. Unfortunately, many of these compounds are poorly tolerated in vivo. The structure of triphenylmethanes indicated a need for conjugated aromatic structures that allow electron delocalization. In order to create similar structures without the triphenylmethane backbone, we synthesized fulvenes through the addition of sodium cyclopentadienide to ketones. Fulvenes are aromatic structures, yet they are highly water soluble. By conjugation of sodium cyclopentadienide with the ketone indigo, we synthesized fulvene-5, which showed inhibitory activity against both Nox4 and Nox2 function. We found that fulvene-5 blocked the production of Ang2 as well as other genes downstream of Nox4, including Nrarp. In addition, fulvene- 5 potently inhibited the growth of bEnd. 3 hemangioma in vivo. This represents what we believe is the first pharmacologic use of any compound with the fulvene structure.

The detailed molecular mechanisms of Nox4 function during hemangioma growth remain to be elucidated. bEnd.3 hemangiomas are a classic model of endothelial cell recruitment in that they grow through a process called host recruitment $(20,21)$. Increased superoxide generation and increased production of Ang 2 may contribute to host recruitment including the recruitment of endothelial precursor cells. Traps devised against the VEGF-induced Notch ligand Dll4 have been produced to address the resistance of tumors to anti-VEGF therapies (22). Blockade of Nox enzymes with small molecules may be an attractive strategy beyond their application in hemangiomas because it may block both Dll4 signaling and superoxide-dependent HIF2 $\alpha$-induced VEGF (23). Combination or sequential therapies using VEGF blockade and Nox 4 blockade using novel small molecules such as fulvenes may result in improved responses for angiogenic disorders including cancer.

\section{Methods}

Cells. bEnd.3 cells (ATCC CRL 2299) were obtained from ATCC and cultured in DMEM (4,500 mg glucose/l) supplemented with 10\% FBS, L-glutamine (14 ml/l), recombinant mouse VEGF (10 ng/ml; R\&D Systems), and antibiotic/antimycotic (14 ml/l; Sigma-Aldrich).
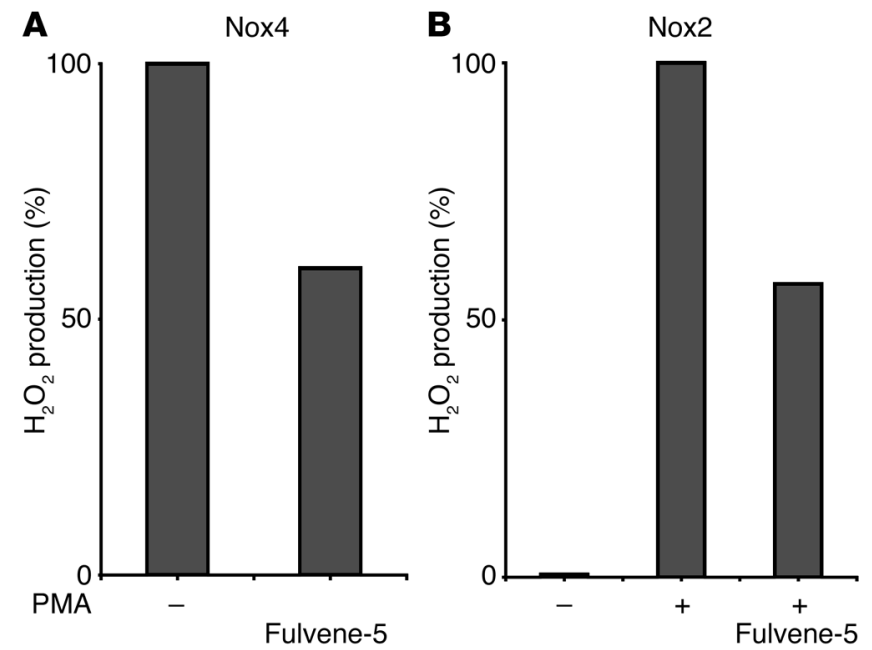

\section{Figure 5}

Fulvene- 5 inhibits ROS generation by Nox 4 and Nox 2 in vitro. 293 cells were treated with vehicle control or $5 \mu \mathrm{M}$ indigo fulvene as described in Methods. COSphox cells were additionally either left unstimulated or stimulated with phorbol ester (PMA). $\mathrm{H}_{2} \mathrm{O}_{2}$ production was measured after 60 minutes. Results are shown as a percentage relative to untreated control $(100 \%)$ and are the mean of $n=4$ (Nox2) or depict a representative experiment (Nox4, $n=2)$. 

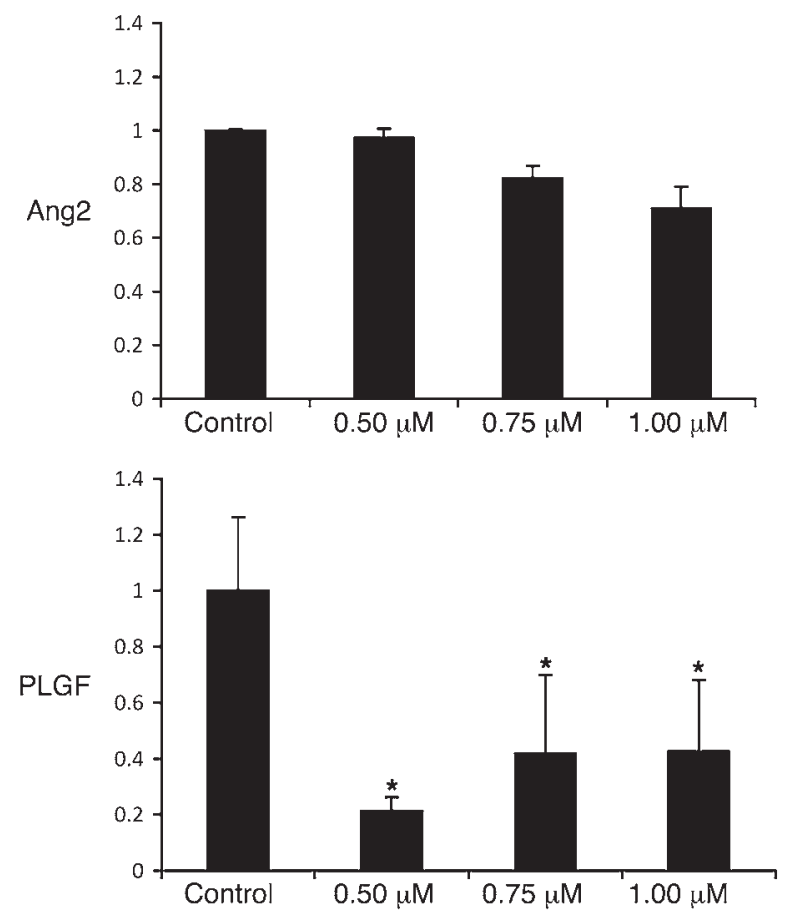
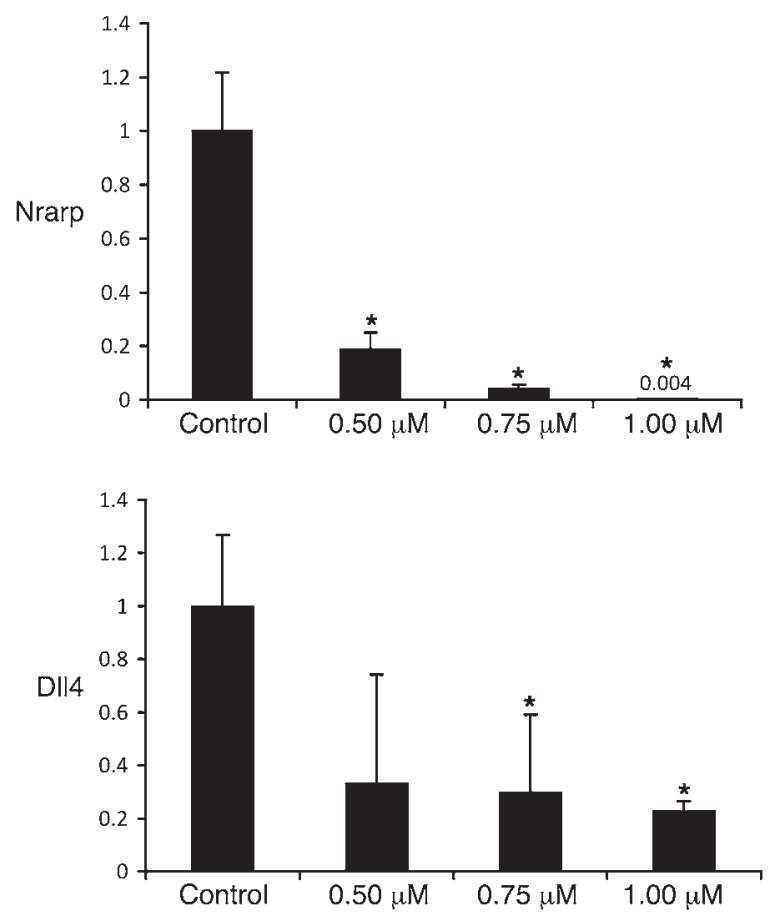

Figure 6

Relative expression levels of Ang2, DII4, and PIGF in bEnd.3 cells treated with fulvene-5. Relative expression levels indicate a dose-dependent decrease. Bars represent the average of triplicate experiments (corrected for $18 \mathrm{~S}$ RNA), and error bars indicate the SEM. ${ }^{\star} P<0.05$ versus control.

Generation of Ang2 $2^{+/+}$, Ang2 $2^{+-}$, and Ang2-/- endothelioma cells. Cerebral capillaries were isolated from Ang $2^{-/-}$mice (24) essentially as described before $(20,21)$, and expression of a known endothelial marker (CD31) was confirmed by PCR. Two independent isolations of endothelial cells and subsequent transformations were used in the experiments. In brief, cortices were dissected free from meninges, minced (in buffer A: $153 \mathrm{mmol} / \mathrm{l} \mathrm{NaCl}$, $5.6 \mathrm{mmol} / \mathrm{l} \mathrm{KCl}, 2.3 \mathrm{mmol} / 1 \mathrm{CaCl}_{2}, 15 \mathrm{mmol} / \mathrm{l} \mathrm{HEPES}$, pH 7.4, $10 \mathrm{mg} / \mathrm{ml}$ $\mathrm{BSA})$, and processed by collagenase digestion ( $0.1 \%$ collagenase II in buffer A; Biochrom) for 30 minutes at $37^{\circ} \mathrm{C}$. Next, brain tissue was pelleted, resuspended in $25 \% \mathrm{BSA}$ in buffer $\mathrm{A}$, and centrifuged at $1,000 \mathrm{~g}$ for 20 minutes at $4^{\circ} \mathrm{C}$. Capillary fragments were cultured overnight at $37^{\circ} \mathrm{C}$ in DMEM (high glucose, with GlutaMAX, sodium pyruvate; Gibco, Invitrogen) supplemented with $10 \% \mathrm{FCS}, 1 \%$ penicillin/streptomycin, $1 \%$ nonessential amino acids, and $5 \times 10^{-5} 2$-mercaptoethanol. Endothelial cell growth supplement (Sigma-Aldrich) was added as a source of growth factors. Endothelioma cell lines from Ang2-deficient (and heterozygous) mice were established the following day by infection with a recombinant retrovirus transducing the polyoma virus middle $\mathrm{T}$ oncogene $(20,21,25)$ as previously described.

Approximately 20,000 endothelioma cells were plated onto $0.2 \%$ gelatincoated coverslips and grown overnight. Cells were washed in PBS and fixed in $4 \%$ paraformaldehyde for 15 minutes and washed again. Antigen blocking was carried out using 10\% FCS for 10 minutes. CD31 was detected using a rat anti-mouse antibody diluted in 5\% FCS (MEC13.3; BD Biosciences) for 1 hour before being washed 3 times. Secondary antibody was an Alexa Fluor 546 conjugated to a donkey anti-rat antibody (1:500 dilution in $5 \%$ FCS; Molecular Probes, Invitrogen), and nuclear costaining was carried out using DAPI $(1: 5,000)$ for 30 minutes. Cells were washed before mounting onto glass slides. All steps were carried out at room temperature.

Lentiviral infection. Two shRNAs targeting the mouse Nox4 mRNA were designed: shNox4-1, GCTGTCCCTAAACGTTCTACT; and shNox4-2, GGGCCTAGGATTGTGTTTA (26). The complementary single-stranded
DNA oligonucleotides were annealed and ligated inside the pENTR/U6 vector according to the manufacturer's instructions (Invitrogen). This vector was recombined by the Gateway LR Clonase (Invitrogen) reaction with the pLenti6/BLOCK-iT-DEST vector (Invitrogen) to form the shRNA-expressing constructs. The lentivirus particles were produced by transient transfection of 293 T cells (27). After 72 hours, the lentivirus-containing supernatant was filtered through $0.45-\mu \mathrm{m}$ pore-sized polyethersulfone membrane and concentrated 150 -fold by ultracentrifugation $(50,000 \mathrm{~g}$ for 90 minutes at $4^{\circ} \mathrm{C}$ ). One day before transduction, bEnd. 3 cells were plated in 12-well plates at a density of 25,000 cells/well. Cells stably expressing shNox4-1 or shNox4-2 were subsequently selected by blasticidin $(7.5 \mu \mathrm{g} / \mathrm{ml})$ for 8 days.

Determination of ROS production. HEK293 cells stably expressing Nox4 or COSphox cells expressing Nox 2 were preincubated with solvent (DMSO) or $5 \mu \mathrm{M}$ indigo fulvene for 15 minutes. $\mathrm{H}_{2} \mathrm{O}_{2}$ release was determined using the homovanillic acid (HVA) assay as described previously (2).

In vivo tumorigenesis studies. In vivo tumorigenesis studies for $\mathrm{Ang}^{+/-}$and Ang ${ }^{-/}$cells were done by injecting 25,000 bEnd.3 cells homozygous for Ang2 and 25,000 bEnd.3 cells heterozygous for Ang2 subcutaneously into 3 mice each. Mice were monitored for the development of tumors and killed 1 month after injection. No evidence of toxicity was observed.

In vivo tumorigenesis studies for bEnd. 3 Nox4shRNA was done by injecting $1 \times 10^{6}$ cells transduced with bEnd. 3 Nox4shRNA and bEnd. 3 control shRNA subcutaneously into 3 nude mice each. Mice were observed for 14 days and then euthanized due to tumor burden in control animals. Tumor measurements were made every other day starting at day 4. Animal studies were approved by the Emory University IACUC.

Quantitative RT-PCR. bEnd.3 $\mathrm{Ang}^{+/-}$cells and bEnd.3 $\mathrm{Ang}^{-/-}$cells were seeded equally into $2 \mathrm{~T}-25$ flasks, and 24 hours later, RNA was extracted and purified using QIAGEN RNeasy Mini Kit (no. 74104) and measured spectrophotometrically (UV-VIS; PerkinElmer). RNA (1 $\mu \mathrm{g})$ was used for DNase amplification (no. 18068-015; Invitrogen) followed by first-strand 


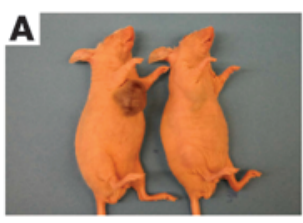

B

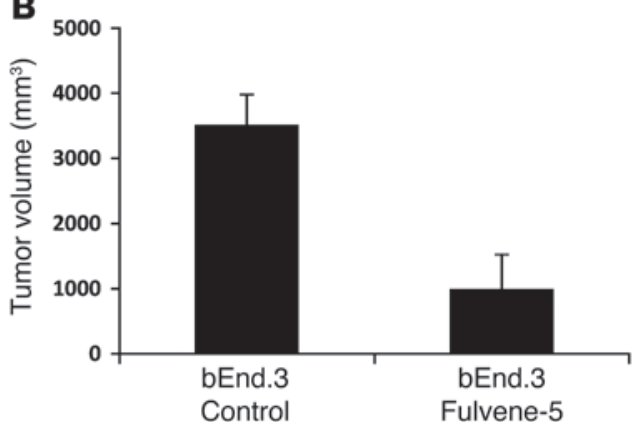

synthesis RT-PCR (no.12371-019; SuperScript, Invitrogen). 96-well Optical Reaction Plate (no. 128; ABI PRISM, Applied Biosystems) was used for the RT-PCR reaction. A measure of $2.5 \mathrm{ml}$ of template, which had been diluted 1:10 in cross-linked water, was used in each well, and the experiment was performed in triplicate. Nrarp (Mm00482529_sl; TaqMan Gene Expression Assay, Applied Biosystems), Nox4 (Mm00479246_ m1; TaqMan Gene Expression Assay, Applied Biosystems) and 18S (Hs99999901_s1; TaqMan Gene Expression Assay, Applied Biosystems) primers were used along with cross-linked molecular grade water (Cellgro; Mediatech Inc.) and master mix (TaqMan Fast Universal PCR Master Mix [2x]; Applied Biosystems). The reaction was run on the 7500 Applied Biosystems Reader for absolute quantification for 96-well plates. Gene expression data were automatically calculated by Sequence Detection Software, version 1.3.1 (Applied Biosystems) along with the SEM. A similar protocol was used for bEnd. 3 Nox4shRNA and bEnd. 3 control cells using Angpt 2 primer (Mm00545822_ml; TaqMan Gene Expression Assay, Applied Biosystems) in addition to Nox4 and 18S. A similar protocol was used for bEnd. 3 cells treated with fulvene- 5 at concentrations of $0.5 \mu \mathrm{M}, 0.75 \mu \mathrm{M}$, and $1.0 \mu \mathrm{M}$ and bEnd. 3 control cells using Nrarp, Dll4 (Mm00444619_m1; TaqMan Gene Expression Assay, Applied Biosystems), PLGF (Mm00435613_m1; TaqMan Gene Expression Assay, Applied Biosystems), Angpt2, and $18 \mathrm{~S}$ primers.

Synthesis and characterization of fulvene-5. Fulvene-5 (Figure 8) was synthesized according to the method of Chajara and Ottosson (28). In brief, $1.0 \mathrm{~g}(3.81 \mathrm{mM})$ of indigo was dissolved in $500 \mathrm{ml}$ tetrahydrofuran (THF) in a clean, dry flask in an argon atmosphere under constant stirring at room temperature. To this, $0.839 \mathrm{~g}(9.53 \mathrm{mM})$ of sodium cyclopentadienide ( $2 \mathrm{M}$ in THF) was added dropwise; upon completion of this addition, the solution was heated to $60^{\circ} \mathrm{C}$ for 4 hours. The solution changed colors from deep blue to blue-green to dark purple and finally to a dark brown. After 4 hours, the reaction was quenched with water and the solvent was removed via rotary

\section{Figure 7}

Treatment with vehicle control and fulvene- 5 in vivo. For each treatment condition, 3 mice were subcutaneously injected on day 1 with $10^{6}$ bEnd. 3 cells and monitored for tumor development. From day 2 to day 12 , mice were treated with fulvene- 5 by making a stock solution of $4 \mathrm{mg}$ fulvene- 5 compound dissolved in $100 \mu \mathrm{l}$ of $100 \%$ ethanol plus $1.1 \mathrm{ml}$ of intralipid and then sonicated for 15 seconds. The treatment stock solution $(330 \mu \mathrm{l})$ was injected intraperitoneally into each of 3 mice of the fulvene- 5 treatment group. For control animals, a stock solution was made of $100 \mu \mathrm{l}$ of $100 \%$ ethanol plus $1.1 \mathrm{ml}$ of intralipid solution, and $330 \mu \mathrm{l}$ of stock solution was injected intraperitoneally into each of 3 mice of the control group. Injections were continued for 2 weeks. On day 9 and day 12, tumors were measured in all 6 animals, and there was a significant difference in tumor volume between the control and fulvene-5-treated mice. No toxicity was noted following injection. Animals were euthanized on day 14 , secondary to tumor burden in the control animals. Photos represent average tumor burden in each of the 2 groups $(\mathbf{A})$, and tumor volume $\left(\mathrm{mm}^{3}\right)$ is graphically depicted $(\mathbf{B})$. Error bars represent SEM. $P<0.05$ versus control.

evaporation at $35^{\circ} \mathrm{C}$. The resulting black plaque-like solid was extracted into diethyl ether until the ether layer was pale yellow. The ether fractions were pooled and evaporated on a rotary evaporator, resulting in a red-orange oil. Chromatographic separation on alumina with 50:50 ether/hexane resulted in a $45 \%$ yield of indigo fulvene in the form of an orange solid. NMR is displayed in Figure 8. The predominant mass spectroscopy point $(\mathrm{M}+)$ is 359.12 .

Statistics. Statistical differences for in vitro assays were done with a 2-tailed Student's $t$ test, as the data from these experiments were normally distributed, with $P<0.05$ considered significant. For the in vivo mouse tumor growth experiment, differences in tumor volume over time were analyzed with 2 -way ANOVA. $P<0.05$ indicated a statistically significant reduction in tumor growth of the treated group compared with the control group.

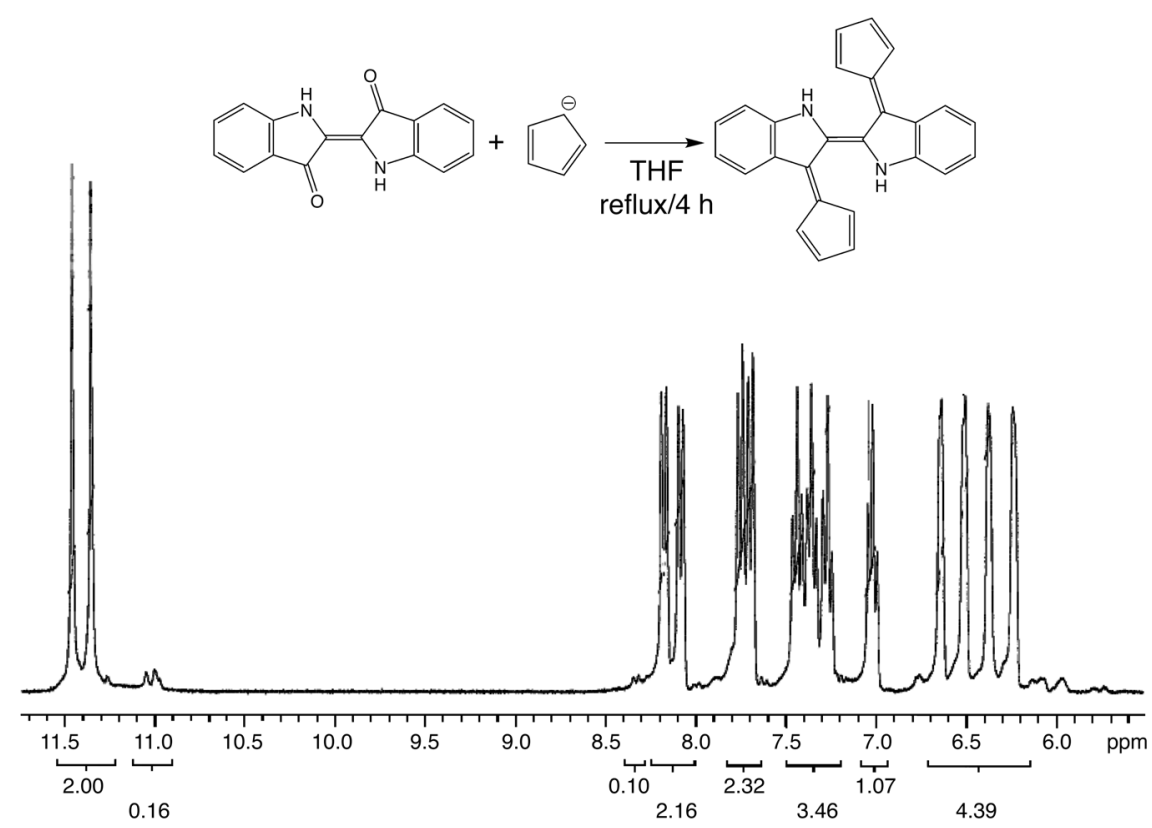

Figure 8

Synthesis and NMR of fulvene-5. Synthesis of fulvene- 5 with indigo and cyclopentadienide. NMR shows clear distinctions of amine peaks characteristic of those found in indigo with added peaks in the aromatic region. THF, tetrahydrofuran. 


\section{Acknowledgments}

J.L. Arbiser was supported by NIH grant R01 AR02030, and grants from the Jamie Rabinowitch-Davis Foundation and the Minsk Foundation. B. Govindarajan was supported by a Dermatology Foundation Career Development award. H.G. Augustin and Y. Reiss were supported by the Deutsche Forschungsgemeinschaft (SFB-TR23, Vascular Differentiation and Remodeling).
Received for publication September 10, 2007, and accepted in revised form May 27, 2009.

Address correspondence to: Jack L. Arbiser, Department of Dermatology, Emory University School of Medicine, WMB 5309, 1639 Pierce Drive, Atlanta, Georgia 30322, USA. Phone: (404) 727-5063; Fax: (404) 727-0923; E-mail: jarbise@emory.edu.
1. Yu, Y., Varughese, J., Brown, L.F., Mulliken, J.B., and Bischoff,J. 2001. Increased Tie2 expression, enhanced response to angiopoietin-1, and dysregulated angiopoietin-2 expression in hemangioma-derived endothelial cells. Am. J. Pathol. 159:2271-2280.

2. Perry, B.N., et al. 2006. Pharmacologic blockade of angiopoietin-2 is efficacious against model hemangiomas in mice. J. Invest Dermatol. 126:2316-2322.

3. Cerimele, F., et al. 2003. Infectious angiogenesis: Bartonella bacilliformis infection results in endothelial production of angiopoetin-2 and epidermal production of vascular endothelial growth factor. Am. J. Pathol. 163:1321-1327.

4. Maisonpierre, P.C., et al. 1997. Angiopoietin-2, a natural antagonist for Tie2 that disrupts in vivo angiogenesis. Science. 277:55-60.

5. Augustin, H.G., Koh, G.Y., Thurston, G., and Alitalo, K. 2009. Control of vascular morphogenesis and homeostasis through the angiopoietin-Tie system. Nat. Rev. Mol. Cell Biol. 10:165-177.

6. Fiedler, U., et al. 2004. The Tie-2 ligand angiopoietin- 2 is stored in and rapidly released upon stimulation from endothelial cell Weibel-Palade bodies. Blood. 103:4150-4156.

7. Stratmann, A., Risau, W., and Plate, K.H. 1998. Cell type-specific expression of angiopoietin-1 and angiopoietin-2 suggests a role in glioblastoma angiogenesis. Am. J. Pathol. 153:1459-1466.

8. Arbiser, J.L., et al. 2002. Reactive oxygen generated by Nox1 triggers the angiogenic switch. Proc. Natl. Acad. Sci. U. S. A. 99:715-720.

9. Bedard, K., and Krause, K.H. 2007. The NOX family of ROS-generating NADPH oxidases: physiology and pathophysiology. Physiol. Rev. 87:245-313.

10. Geiszt, M. 2006. NADPH oxidases: new kids on the block. Cardiovasc. Res. 71:289-299.

11. Kawahara, B.T., Quinn, M.T., and Lambeth, J.D. 2007. Molecular evolution of the reactive oxygengenerating NADPH oxidase (Nox/Duox) family of enzymes. BMC Evol. Biol. 7:109.

12. Kwon, J., et al. 2004. Reversible oxidation and inactivation of the tumor suppressor PTEN in cells stimulated with peptide growth factors. Proc. Natl. Acad. Sci. U. S. A. 101:16419-16424.

13. Yu, L., Zhen, L., and Dinauer, M.C. 1997. Biosynthesis of the phagocyte NADPH oxidase cytochrome b558. Role of heme incorporation and heterodimer formation in maturation and stability of gp91phox and p22phox subunits. J. Biol. Chem. 272:27288-27294.

14. Dinauer, M.C., Orkin, S.H., Brown, R., Jesaitis, A.J., and Parkos, C.A. 1987. The glycoprotein encoded by the $\mathrm{X}$-linked chronic granulomatous disease locus is a component of the neutrophil cytochrome b complex. Nature. 327:717-720.

15. Parkos, C.A., Dinauer, M.C., Jesaitis, A.J., Orkin, S.H., and Curnutte, J.T. 1989. Absence of both the $91 \mathrm{kD}$ and $22 \mathrm{kD}$ subunits of human neutrophil cytochrome $\mathrm{b}$ in two genetic forms of chronic granulomatous disease. Blood. 73:1416-1420.

16. Brar, S.S., et al. 2001. Reactive oxygen species from $\mathrm{NAD}(\mathrm{P}) \mathrm{H}$ :quinone oxidoreductase constitutively activate NF-kappaB in malignant melanoma cells. Am. J. Physiol. Cell Physiol. 280:C659-C676.

17. Govindarajan, B., et al. 2007. Overexpression of Akt converts radial growth melanoma to vertical growth melanoma. J. Clin. Invest. 117:719-729.

18. Lapidoth, M., et al. 2009. Efficacy of topical application of eosin for ulcerated hemangiomas. J. Am. Acad. Dermatol. 60:350-351.
19. Lodha, S., et al. 2002. Angiotensin II-induced mesangial cell apoptosis: role of oxidative stress. Mol. Med. 8:830-840.

20. Bautch, V.L., Toda, S., Hassell, J.A., and Hanahan, D. 1987. Endothelial cell tumors develop in transgenic mice carrying polyoma virus middle $\mathrm{T}$ oncogene. Cell. 51:529-537.

21. Williams, R.L., et al. 1989. Endothelioma cells expressing the polyoma middle $\mathrm{T}$ oncogene induce hemangiomas by host cell recruitment. Cell. 57:1053-1063.

22. Noguera-Troise, I., et al. 2006. Blockade of Dll4 inhibits tumour growth by promoting non-productive angiogenesis. Nature. 444:1032-1037.

23. Maranchie, J.K., and Zhan, Y. 2005. Nox4 is critical for hypoxia-inducible factor 2-alpha transcriptional activity in von Hippel-Lindau-deficient renal cell carcinoma. Cancer Res. 65:9190-9193.

24. Gale, N.W., et al. 2002. Angiopoietin-2 is required for postnatal angiogenesis and lymphatic patterning, and only the latter role is rescued by Angiopoietin-1. Dev. Cell. 3:411-423.

25. Kiefer, F., et al. 1994. Endothelial cell transformation by polyomavirus middle $\mathrm{T}$ antigen in mice lacking Src-related kinases. Curr. Biol. 4:100-109.

26. Li, J., et al. 2006. The NADPH oxidase NOX4 drives cardiac differentiation: role in regulating cardiac transcription factors and MAP kinase activation. Mol. Biol. Cell. 17:3978-3988.

27. Dull, T., et al. 1998. A third-generation lentivirus vector with a conditional packaging system. J. Virol. 72:8463-8471.

28. Chajara, K., and Ottosson, H. 2004. An improved pathway to 6,6-disubstituted fulvenes. Tetrahedron Lett. 45:6741-6744. 